\title{
Implementation of Signal Detection and Control of Bioartificial Liver Support System
}

\author{
Changzhe $\mathrm{Wu}$ \\ Beijing Key Laboratory of \\ Bioelectromagnetism, Institute of \\ Electrical Engineering, Chinese \\ Academy of Sciences, University of \\ Chinese Academy of Sciences, Beijing, \\ China \\ wuzhe@mail.iee.ac.cn
}

\author{
$\mathrm{Ke} \mathrm{Li}$ \\ University of Chinese Academy of \\ Sciences, Beijing, China \\ like@mail.iee.ac.cn
}

\author{
Cheng Zhang \\ Beijing Key Laboratory of \\ Bioelectromagnetism, Institute of \\ Electrical Engineering, Chinese \\ Academy of Sciences, University of \\ Chinese Academy of Sciences, Beijing, \\ China \\ zhangchengcc@mail.iee.ac.cn
}

\author{
Guanghao Zhang \\ Beijing Key Laboratory of \\ Bioelectromagnetism, Institute of \\ Electrical Engineering, Chinese \\ Academy of Sciences, University of \\ Chinese Academy of Sciences, Beijing, \\ China \\ zhangguanghao@mail.iee.ac.cn
}

\author{
Xiaolin Huo* \\ Beijing Key Laboratory of \\ Bioelectromagnetism, Institute of \\ Electrical Engineering, Chinese \\ Academy of Sciences, University of \\ Chinese Academy of Sciences, Beijing, \\ China \\ huoxl@mail.iee.ac.cn
}

\section{CCS CONCEPTS}

\begin{abstract}
Liver failure is an extremely dangerous disease whose mortality rate is $73.9 \%$ and the rate of acute liver failure is as high as $87.8 \%$. As the only effective way to treat liver failure, liver transportation is facing a serious shortage of donor. As an extracorporeal circulation device, artificial liver support system could effectively replace the patient's liver in a short period of time so as to improve the survival rate of patients greatly. At present, the control core of the artificial liver control system is PLC. However, with the increasing complexity of functions and demands, the control system based on PLC will gradually show some disadvantages in cost, safety, reliability, stability, real-time Performance and other aspects which will restrict the development of artificial liver. Aiming at the deficiencies in the existing control system, a bio-artificial embedded control system based on ARM and CPLD was designed and implemented in this study, completes the hardware design, PCB design and control program writing, and designs the safety control loop to improve the control system Reliability. The experimental results show that performance of the control system has been improved to a certain extent in terms of safety, reliability, stability and real-time performance. Meanwhile, the cost of control system is lower, which is conducive to the market promotion of artificial liver.
\end{abstract}

Permission to make digital or hard copies of all or part of this work for personal or classroom use is granted without fee provided that copies are not made or distributed for profit or commercial advantage and that copies bear this notice and the full citation on the first page. Copyrights for components of this work owned by others than ACM must be honored. Abstracting with credit is permitted. To copy otherwise, or republish, to post on servers or to redistribute to lists, requires prior specific permission and/or a fee. Request permissions from permissions@acm.org.

BIBE2021, fuly 20-22, 2021, Hangzhou, China

(C) 2021 Association for Computing Machinery.

ACM ISBN 978-1-4503-8929-7/21/07 . \$15.00

https://doi.org/10.1145/3469678.3469703
- Applied computing; • Life and medical sciences;

\section{KEYWORDS}

Bioartificial liver, Signal detection, Embedded Control, ARM, CPLD

\section{ACM Reference Format:}

Changzhe Wu, Ke Li, Cheng Zhang, Guanghao Zhang, and Xiaolin Huo*. 2021. Implementation of Signal Detection and Control of Bioartificial Liver Support System. In The Fifth International Conference on Biological Information and Biomedical Engineering (BIBE2021), fuly 20-22, 2021, Hangzhou, China. ACM, New York, NY, USA, 7 pages. https://doi.org/10.1145/3469678. 3469703

\section{INTRODUCTION}

As one of the most important organs of human body, the liver has functions of synthesis, detoxification, metabolism, secretion, biological transformation and immune defense. However, when the liver is severely damaged by a variety of factors, (such as virus, alcohol, drugs, etc.), it will cause massive necrosis of liver cell, lead to serious liver dysfunction, and then develop into liver failure. The mortality rate of liver failure is as high as $73.9 \%$, among which the mortality rate of acute liver failure is as high as $87.8 \%$ [1]. At present, the only effective way to treat liver failure is liver transplantation. However, the primary problem of liver transplantation is lack of donor, which severely restricts the clinical application of liver transplantation [2]. More than 300,000 people in our country desperately need liver transplants every year to save lives. However, only a few thousands of people have received the transplant successfully, $50 \%$ of the remaining receptors died within a year [3]. This contradiction between supply and demand makes liver transplantation difficult to become the main therapeutic approach for liver failure in the short term. 


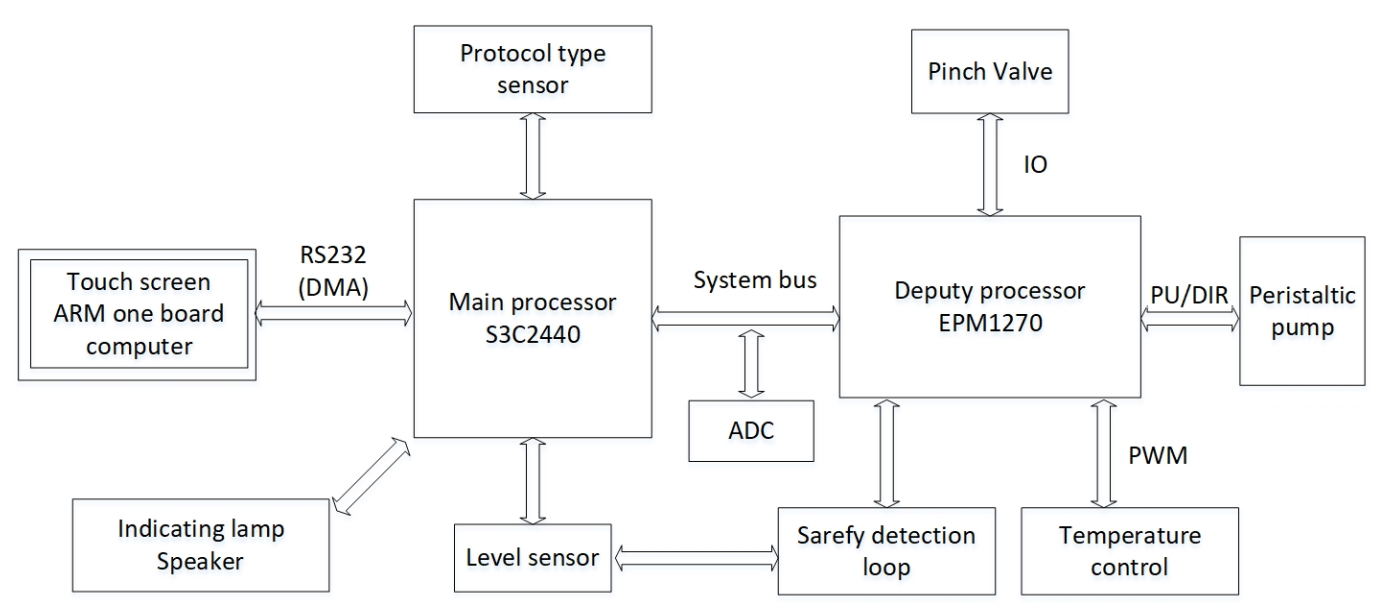

Figure 1: Topology of Control System.

The artificial liver support system uses extracorporeal circulation to draw blood that should normally flow into the human liver to the artificial live, and when the blood flows through the artificial liver, it is returned to the body. Thus, the purpose of using artificial liver to replace the patient's liver for a short time is achieved. Nowadays, artificial liver treatment has been widely used in the treatment of liver failure [4, 5]. At present, different types of artificial liver have entered the phase I to III clinical [6], mainly including HepatAssist 2000 [7], ELAD [8], BLSS AMC-BAL [9], Hybrid LSS-MELS systems, etc [10]. The core controller of the artificial liver control system is mostly PLC(programmable logic controller), The control system with PLC control core has the characteristics of simple programming, strong adaptability, and easy to build a platform [11].However, with the increasing complexity of the artificial liver function, the increasing demand in control requirement, the PLC system with modular control will gradually show some disadvantages in terms of cost, safety, reliability, stability, real-time Performance and volume of control unit, which limits the development of artificial liver. For this reason, a new artificial liver system control scheme is proposed in this paper, the function of artificial liver is realized based on ARM and CPLD, and the performance optimization is carried out for the deficiency of existing control system.

\section{CONSTRUCTION OF HARDWARE PLATFORM}

Artificial liver control system mainly includes controller, humanmachine interface, peristaltic pump, heparin pump, valve, fastener, protocol communication type sensor, level sensor, heating plate, alarm indicator, solenoid valve, safety circuit, etc. The topology structure of the control system is shown in Figure 1. Among them, the main processor adopts ARM9 kernel S3C2440, the main task of which is to control the scheduling of the system, detect and calculation processing System sensor data, issue peripheral control module control instruction, control alarm system signal; the secondary treatment adopts EPM1270, the main task of which is to control peristaltic pump, heparin pump, pinch valve, fastener, heating plate and other devices; AD7606 is a 16-bit bipolar AD acquisition chip, which mainly used for the detection of heparin pump position and data collection of PT100 temperature sensor.

The peristaltic pump actuator adopts YZ-ACSD608, the motor of it is $24 \mathrm{~V}$ to three-phase AC motors, and pulse + direction control mode is adopted; the clamp valve and lock driver adopt DKC-230A, its motor is stepping motor, and it adopts point control mode; heparin pump drive adopts SMD-202M, the motor is stepping motor, and pulse + direction control mode is adopted; heating plate adopts heater band with $24 \mathrm{~V}$ and $100 \mathrm{~W}$, its sensor adopts PT100 that precision is $0.1^{\circ} \mathrm{C}$.

\subsection{Communication Between Modules}

In the design of the control system, we first treat the EPM1270 and AD7606 as storage with high bandwidth external storage interfaces, and then extend EPM1270 and AD7606 to Bank2 and Bank3 respectively in S3C2440, the starting address is set to $0 \times 10,000,000$ and $0 \times 18,000,000$. In the end, extend 16-bit system bus through hardware, in order to achieve the communication between S3C2440, EPM1270 and AD7606. The connection mode is shown in Figure 2

Main controller connects with touch screen and protocol type sensor by RS-232 and RS-485, The agreement voltage conversion chip is used for MAX3232 and MAX485 respectively. The communication between the main controller and the touch screen is designed with the identification code and the CRC test code. MODBUS protocol communication is used between master control and protocol type sensor.

\subsection{Acquisition Circuit of Heating Plate Temperature}

The heating plate temperature sensor used in artificial liver is PT100 platinum resistance temperature sensor, precision of which is $0.1^{\circ} \mathrm{C}$, It is mainly used to feed the heating plate temperature to achieve the purpose of providing stable temperature environment for circulating plasma and biological reaction tank. The heating plate temperature acquisition circuit first collects the voltage value of PT100, In turn, after amplifying the voltage signal, the signal is 


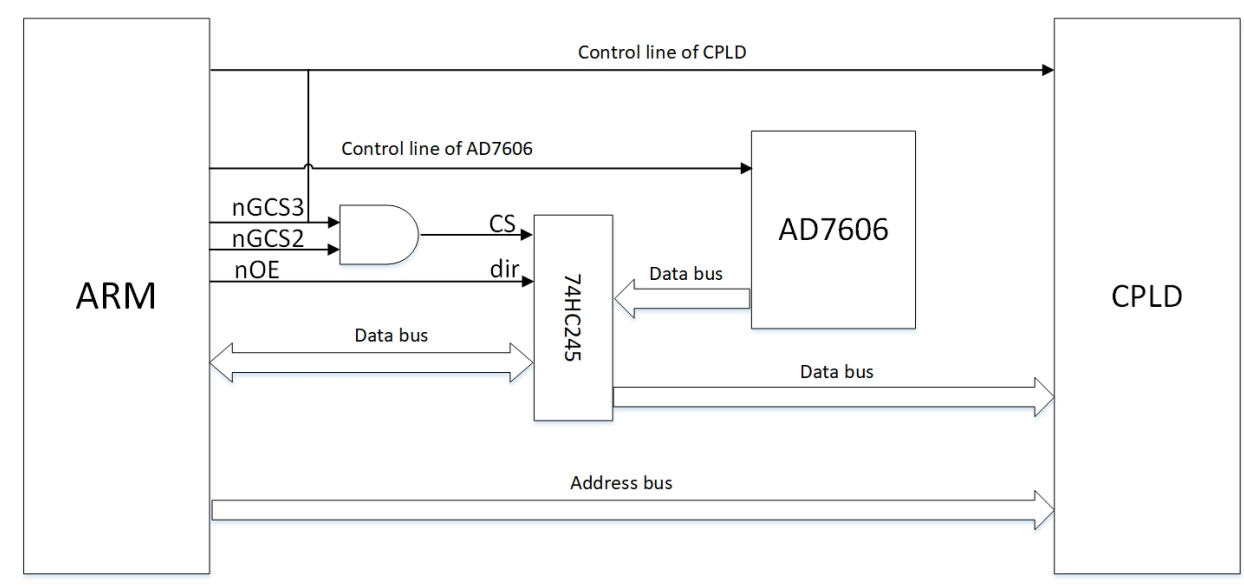

Figure 2: Block Diagram of System Bus Connection.

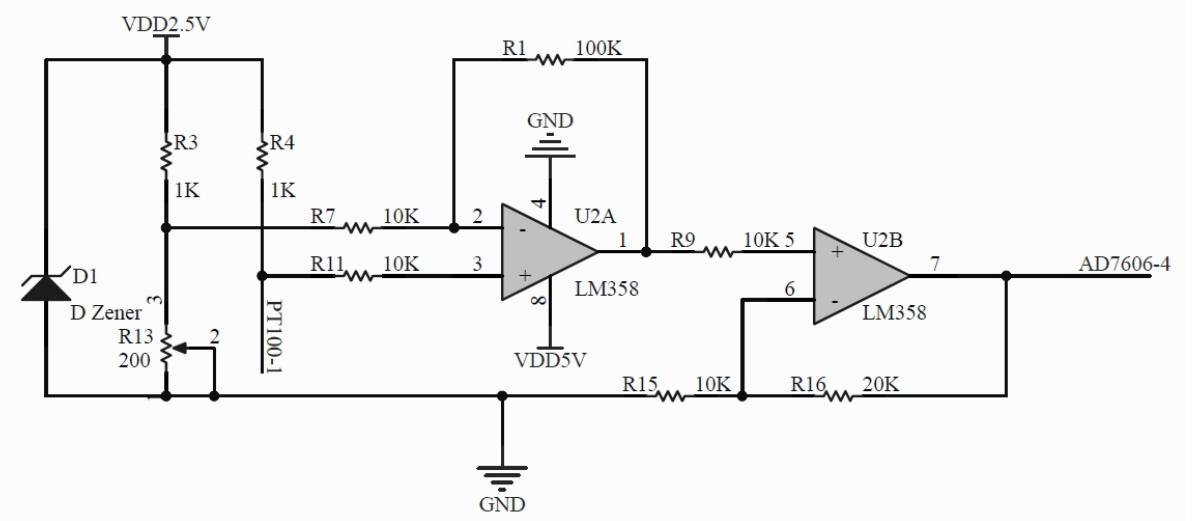

Figure 3: Schematic Diagram of Preamplifier Circuit.

converted with $\mathrm{AD}$, the data is converted to temperature, and the temperature data is read in the main controller. The preamplifier circuit is shown in Figure 3. The amplification circuit adopts single - power supply for LM358. In order to improve the linearity of amplifying circuit, a two-stage amplification circuit is adopted, The first level amplifies the small signal collected, and the magnification is 11 , magnification of the second stage is 3 , a total of 33. After amplification, the signal is converted by AD7606 channel 4. By adjusting the adjustable resistor of $200 \Omega$, amplifying circuit for zero point correction, improving the accuracy of amplifying circuit.

\subsection{Design of Safety Circuit}

In the control system, the main controller can monitor bubbles, liquid level and blood supply shortage sensors through external interruption, When the state of the sensor is alarm, it enters the safety protection program to perform the operation of the peristaltic pump to ensure the patient's safety. However, when the controller fails to respond to the alarm interrupt signal in time, the peristaltic pump cannot be suspended in time to bring danger to the patient. Therefore, a separate hardware safety circuit is set to avoid the above situation. The safety circuit is shown in Figure 4 .
The enable end of the peristaltic pump operation is formed by the combination of the main controller and the sensor level series signal through the AND gate.. When the sensor sends an alarm signal, in order to provide the main controller with the appropriate time to respond to the interrupt, the hardware signal after the serial connection will act on the peristaltic pump enable terminal after a delay of about 1.1s. In this way, it avoids the system reliability due to too sensitive safety circuit.

The delay circuit is shown in Figure 5. With the 555 timer, the output control signal is changed as the capacitor $\mathrm{C} 1$ voltage increases and the resistance R21 voltage changes to about $1.66 \mathrm{~V}$. Resistance R21 voltage varies with time as in formula (1), when $\mathrm{C} 1=100 \mathrm{uf}, \mathrm{R} 21=10 \mathrm{k} \Omega$, delay time is about $\mathrm{T} \approx 1.1 \times \tau=1.1 \times \mathrm{RC}=1.1 \mathrm{~s}$.

$$
U_{r}=U_{\text {alarm }}\left(1-e^{-t / R C}\right.
$$

\section{SOFTWARE PROGRAMMING}

The design of artificial liver software includes the design of the upper computer monitor screen and the control program of the lower computer. The upper computer adopts LABVIEW to design 


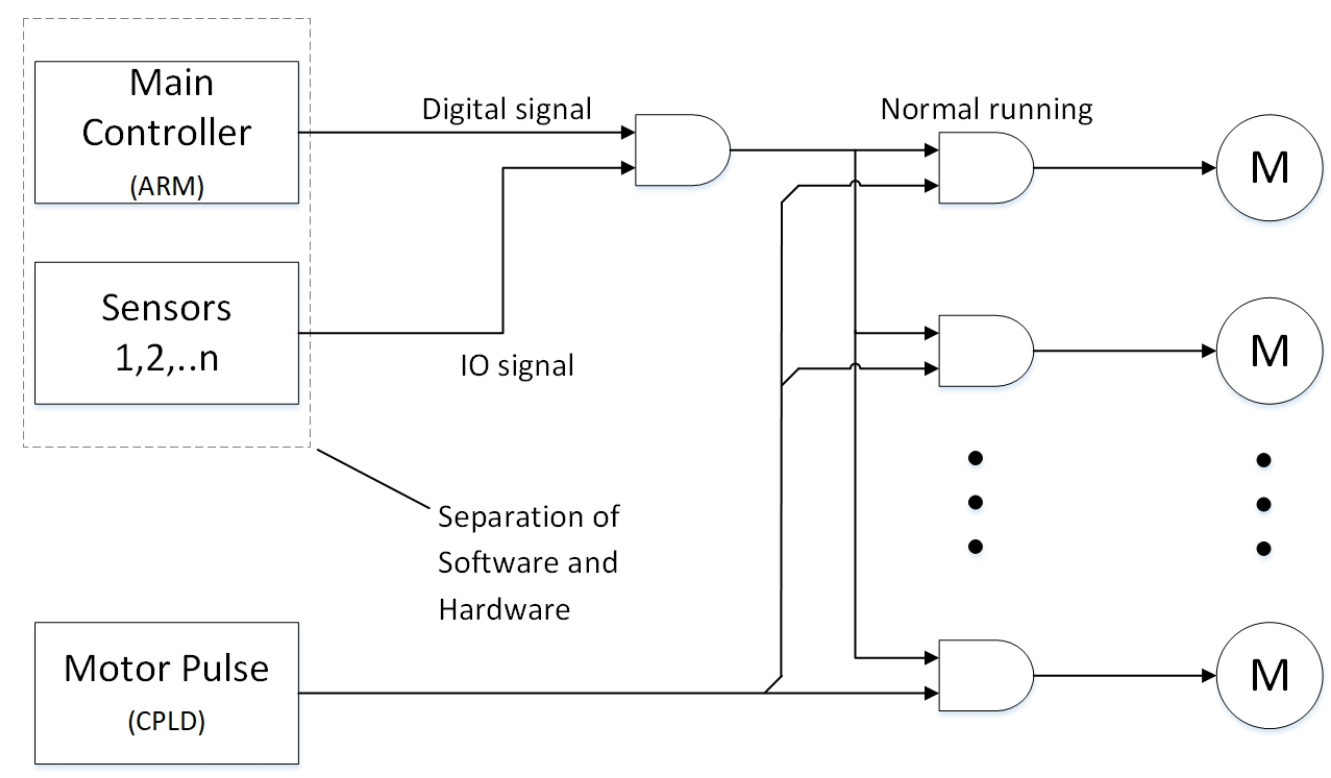

Figure 4: Schematic Diagram of Safety Circuit.

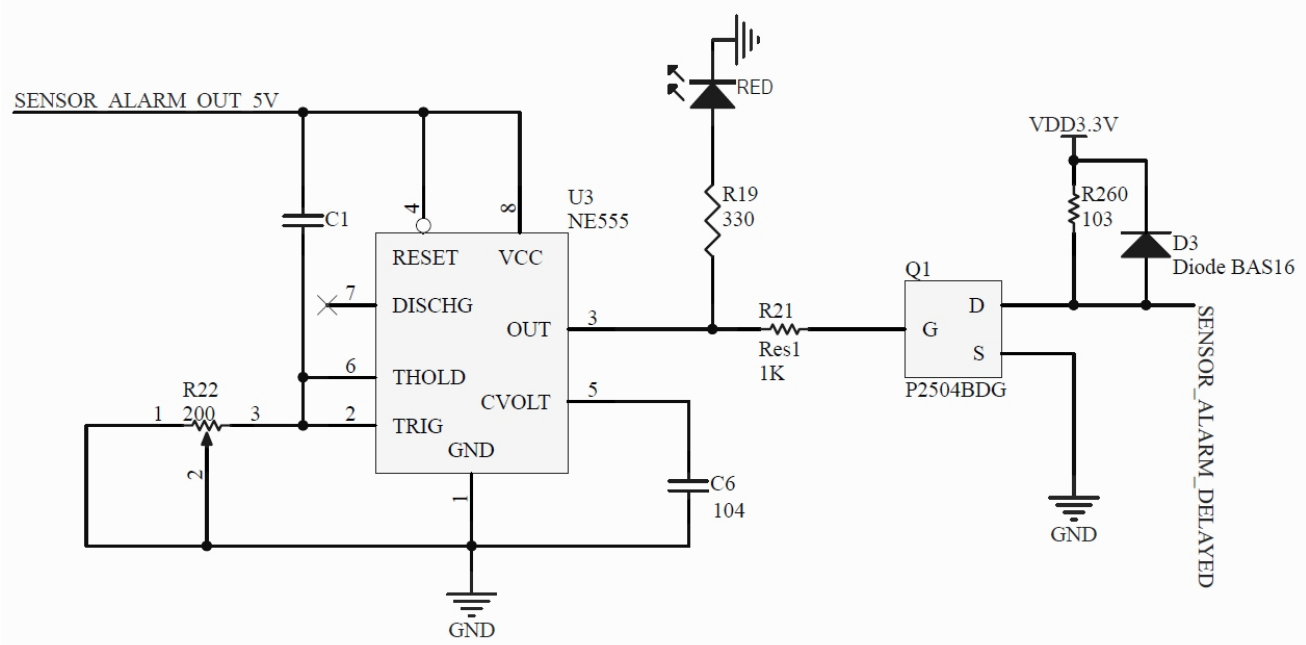

Figure 5: Schematic Diagram of Alarm Signal Delay Circuit.

the monitoring interface, and implement the command, data transmission and data reading. The lower computer realizes control and detection of peristaltic pump, clamp valve, heparin pump, sensor, heating plate and so on.

\subsection{Control Algorithm of Peristaltic Pump and Heparin Pump}

The peristaltic pump in the artificial liver adopts a pulse + direction control method. The main controller S3C2440 achieves the purpose of controlling the different frequency pulse of EPM1270 by adjusting the frequency count comparison of the secondary controller
EPM1270 memory, then the control of the rotational speed is realized. The memory bandwidth is 16 bits and the highest position is the direction control, 1 indicates that the peristaltic pump rotates in the forward direction, and 0 indicates that the peristaltic pump rotate in the reverse direction; the lower 15 bits represent the size of the divider. Among them, the calculation formula of frequency counting is shown in formula (2) :

$$
C=\frac{50000000}{2 K_{a}} \times \frac{1}{2 \times P_{a} \times Q}
$$

50,000,000 represents the EPM1270 clock frequency; Ka represents the division frequency coefficient of EPM127; Pa Indicates the pulse frequency required when the peristaltic pump flow is set to $1 \mathrm{ml} / \mathrm{min}$; 
$Q$ represents the amount of traffic to be set. The control parameters of the creep pump are set as follows: $\mathrm{Ka}=3, \mathrm{~Pa}=60$; the control parameters of heparin pump are as follows: $\mathrm{Ka}=60, \mathrm{~Pa}=3.19$. The value of the divider is 15 bits, so the range of data is $0 \sim 32,737$, according to the formula (2), the minimum flow rate of the peristaltic pump can be approximately $2.2 \mathrm{ml} / \mathrm{min}$; the minimum flow of heparin pump can be approximately $1 \mathrm{ml} / \mathrm{h}$, all can satisfy the system requirement.

\subsection{Rotation Pump Control Algorithm}

The artificial liver rotary pump also adopts pulse + direction control. However, the data that needs to be set during operation is the rotation period, which controls $180^{\circ}$ periodic operation of the rotary pump. The calculation of the numerical value of the rotary pump divider is as the formula (3) :

$$
C=\frac{50000000}{2 K_{a}} \times \frac{1}{2 \times f}
$$

$\mathrm{Ka}=3$; $\mathrm{f}$ Indicates the frequency of the pulse that needs to be set. The directional switching limit signal of rotary pump is provided by three photoelectric switches. When the rotation is in place, the photoelectric switch provides the signal and the rotation direction is switched in EPM1270. The rotation period and pulse frequency are not linear due to the delay of the switching instantaneous. The calculation formula of the pulse control frequency and the rotation cycle of the rotary pump are obtained through the measurement and the segmentation of the data, as shown in formula (4):

$$
f=\left\{\begin{array}{c}
-34.24 \times T+1712.2(20<T \leq 30) \\
-102.73 \times T+3082(10<T \leq 20) \\
-408.26 \times T+6137.3(5<T \leq 10) \\
-4095.95 \times T+24590.75(1<T \leq 5)
\end{array}\right.
$$

\subsection{Program of Sensor Data Reading}

When the artificial liver system is in operation, it is necessary to constantly collect the data of the sensor to monitor the normal operation of the instrument. The detection process is shown in Figure 6

The system adopts MODBUS protocol, which reads the data of pressure, weighing, $\mathrm{PH}$, DO, blood leakage and other sensors in the form of polling. The process of reading instruction send and state judgment are implemented in the timer interrupt, the process of reading instruction send and state judgment is implemented in the timer interrupt. When a sensor does not respond to a system's read command for three consecutive times, it is determined that the sensor is running abnormally, and the ALARM_REMIND status is sent to the monitor screen and the yellow alarm indicator light is lit.

\subsection{Interrupt Priority Setting}

There are 12 external interrupts and 2 timer interrupts in artificial liver control. The safety, real time, and reliability of the system operation can be guaranteed only by the proper allocation of interrupted priorities. According to the speed and importance of corresponding function to each interrupt in the artificial liver, the priority of the configuration system interrupt is shown in Table 1 from high to low:
By configuring the register PRIORITY of S3C2440, the PRIORITY of each interrupt signal in ARB_SEL6 is setting as 0-2-3-1-5, and the priority rotation enable of ARBBITER6 is turned off to realize the configuration as shown in the table.

\section{EXPERIMENTAL RESULTS AND ANALYSIS}

Debug the control panel installed on the design. Experiments mainly aimed at the peristaltic pump flow, heparin pump flow, rotary pump cycle, the heating plate temperature control, electromagnetic valve on-off, clip pipe valve switch, protocol sensor communication, level sensor alarm, blood pump and rotary pump speed control, safety circuit etc.

During the experiment, when peristaltic pump flow was set to $5 \mathrm{ml} / \mathrm{min}, 50 \mathrm{ml} / \mathrm{min}, 100 \mathrm{ml} / \mathrm{min}, 200 \mathrm{ml} / \mathrm{min}$ and $250 \mathrm{ml} / \mathrm{min}$, the error remains within the range of $-4.7 \% \sim+4.71 \%$, which meets the requirements of the system $-5 \% \sim+10 \%$; when the flow of heparin pump was set at $1 \mathrm{ml} / \mathrm{h}, 10 \mathrm{ml} / \mathrm{h}$ and $20 \mathrm{ml} / \mathrm{h}$ respectively, the error remains at $-4.6 \% \sim+0.2 \%$, which satisfies the requirements of the system- $5 \% \sim+5 \%$; when the rotary pump cycle was set to $18 \mathrm{~s}, 20 \mathrm{~s}$, $22 \mathrm{~s}, 24 \mathrm{~s}, 26 \mathrm{~s}, 28 \mathrm{~s}$ and $30 \mathrm{~s}$ respectively, the error remains at $-0.25 \% \sim+$ $5.11 \%$, which meets the requirements of the system $-10 \% \sim+10 \%$; when the flow of blood pump is $50 \mathrm{ml} / \mathrm{min}$, the heating plate temperature was setting to $35^{\circ} \mathrm{C}, 36^{\circ} \mathrm{C}$ and $37^{\circ} \mathrm{Crespectively,} \mathrm{heated} \mathrm{liquid}$ temperature fluctuations in value plus or minus $0.1^{\circ} \mathrm{C}$ scope, meet the system requirements; The safety circuit is tested by turning off the sensor in S3C2440, When the sensor alarms, the peristaltic pump stops rotating about $1 \mathrm{~s}$ later The function of the safety circuit meets the design requirements; Under the condition ofnormal operation of the artificial liver components, the fatigue test was carried out for 4 consecutive days. During the experiment, the system was operating stably withoutfailure.

\section{CONCLUSION}

Based on the deficiencies of existing artificial liver control system in terms of cost, safety, reliability, stability, real time, scalability etc., this paper proposes a brand-new biological artificial liver embedded control system based on ARM and CPLD. The main control circuit and peripheral module circuit are designed and implemented, and corresponding control motheds and algorithms are designed for each module, and finally installed for testing. After testing, its safety, reliability, stability real-time, and other aspects are correspondingly improved compared with the existing artificial liver system, and the control accuracy meets the system requirements. The control system based on ARM and CPLD greatly reduces the cost of control unit, which brings benefits to the promotion and industrialization of artificial liver systems. At the same time, the control unit is smaller in size, which is more conducive to optimizing the mechanical structure of artificial liver, and brings convenience to the overall appearance of the artificial liver system and system writing.

\section{ACKNOWLEDGMENTS}

This study was supported by the National Key R\&D Program of China(2018YFC1106400, 2018YFA0108200), the Natural Science Foundation of China(Grant No. 61671428). 


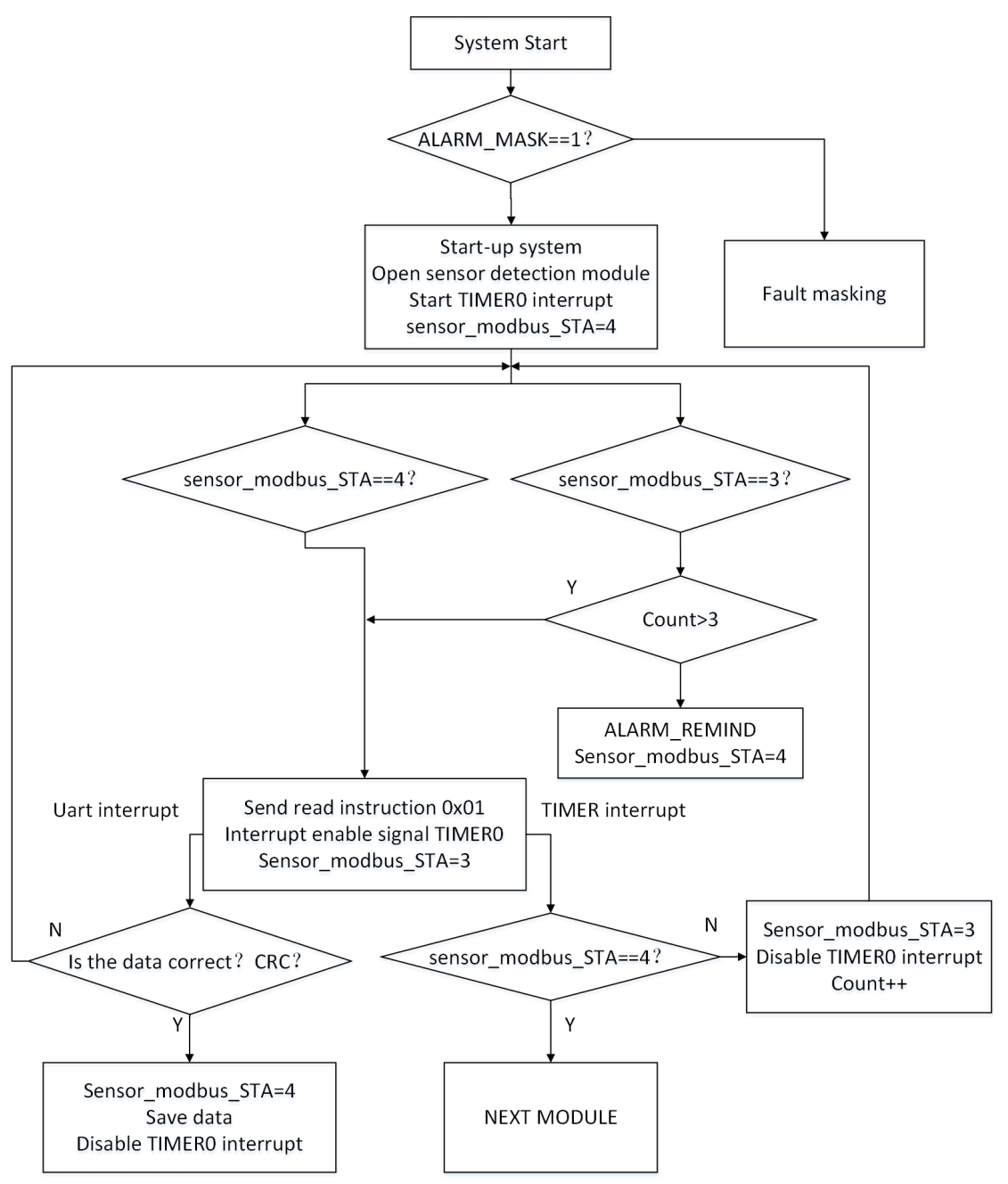

Figure 6: Block Diagram of Sensor Detection.

Table 1: The Table of Interrupt Priority Configuration.

\begin{tabular}{lll}
\hline Interrupt group & Interrupt & System function \\
\hline ARBBITER0 & EINT_0 & Insufficient blood supply sensor alarm \\
& EINT_1 & Bubble sensor alarm \\
EINT_2 & Liquid level sensor alarm \\
ARBBITER2 & uart2 reception & The main control chip receives the touch screen command \\
ARBBITER3 & DMA & The main control chip sends data to the touch screen \\
ARBBITER4 & uart1 reception & Modbus protocol sensor receive data \\
ARBBITER1 & EINT_4 & Increase blood pump flow \\
& EINT_5 & Reduce blood pump flow \\
& EINT_6 & Increase cycle pump cycle \\
& EINT_7 & Reduce cycle pump cycle \\
& EINT_9 & The AD7606 transform is completed \\
& EINT_18 & Send sensor reading instructions through uart1 \\
\hline
\end{tabular}




\section{REFERENCES}

[1] Zongcheng Ge, Huiyang Xu, Weishun He, et al. (2009) The analysis of clinical characteristics of different types of liver failure and its related risk factors [J]. Journal of Clinical Hepatology, 25(5), 352-354.

[2] Stravitz RT, Kramer AH, Davern T, et al. (2007) Acute liver failure study group. Intensive care of patients with acute liver failure: recommendations of the U.S Acute liver failure study group[J]. Crit Care Med, 35(11), 498-2508.

[3] Yifeng Tao, Zhecheng Zhu, Chenghong Peng. (2014) The opportunity and challenge of DCD donor liver transplantation in China [J]. Journal of Surgery Concepts \& Practice, 19(4), 277-280.

[4] Jaime M Glorioso, Shennen A Mao, Brian Rodysill, et al. (2015) Pivotal preclinical trial of the spheroid reservoir bioartificial liver[J]. Journal of Hepatology, (63), 388-398.

[5] Hassanein Tarek I, Schade Robert R, Hepburn Iryna S. (2011) Acute-on-chronic liver failure: extracorporeal liver assist devices[J]. Curr Opin Crit Care, 17(2),195203.

[6] Meimei Duan, Xiaolin Huo ,Changzhe Wu, et al. (2011) The research progress of dissolved oxygen control in biological artificial liver support system [J]. Chinese Journal of Biomedical Engineering, 30(3), 462-467.

[7] Sauer IM, Kardassis D, Zeillinger K, et al. (2003) Clinical extracoproreal hybrid liver support-phase I study with primary porcine liver cells[J]. Xenotransplantation, 10(5), 160-469.

[8] Pless G, Sauer IM. (2005) Bioartificial liver :current status[J]. Transplant Proc, 37(9), 3893-3895.

[9] Mazariegos GV, Patzer JF, 2nd, Lopez RC, et al. (2002) First clinical use of a novel bioartificial liver support system(BLSS)[J]. Am J Transplant, 2(3), 260-266.

[10] Gerlach JC. (2006) Bioreactors for extracorporeal liver support[J]. Cell Transplant, 15,1_suppl, 91-103.

[11] Xiantao Shi. (2014) Characteristics of PLC and its application in electrical automation system[J]. Digital Technology and Application , (07),11-12.f 